Article

\title{
The Reentrant Four-Layer Quasi-Elliptic Bandstop Filter
}

\author{
Victor V. Atuchin ${ }^{1,2,3, *}$, Anatoly P. Gorbachev ${ }^{4}$, Vladimir A. Khrustalev ${ }^{5}$ and \\ Natalya V. Tarasenko ${ }^{6}$ \\ 1 Laboratory of Optical Materials and Structures, Institute of Semiconductor Physics, SB RAS, \\ Novosibirsk 630090, Russia \\ 2 Laboratory of Semiconductor and Dielectric Materials, Novosibirsk State University, \\ Novosibirsk 630090, Russia \\ 3 Research and Development Department, Kemerovo State University, Kemerovo 650000, Russia \\ 4 Radio-Receiving and Radio-Transmitting Devices Department, Novosibirsk State Technical University, \\ Novosibirsk 630073, Russia; apgor@ngs.ru \\ 5 Department of Electronic Devices, Novosibirsk State Technical University, Novosibirsk 630073, Russia; \\ va_khrustalev@ngs.ru \\ 6 General Physics Department, Novosibirsk State Technical University, Novosibirsk 630073, Russia; \\ natalyavtarasenko@gmail.com \\ * Correspondence: atuchin@isp.nsc.ru
}

Received: 5 December 2018; Accepted: 2 January 2019; Published: 10 January 2019

\begin{abstract}
The novel microwave quasi-elliptic bandstop filter, which uses the initially uncoupled strip transmission lines, is considered in this work. The proposed filter is based on the reentrant structure, where the metallic body with a floating potential is asymmetric. Generally speaking, the internal and external dielectric fillings must have their unequal relative permittivities. As a result, additional symmetrical reflection zeros are reached at the lower and upper pass bands of the stop band leading to the quasi-elliptic function response that improves the filter selectivity. The general transverse electromagnetic (TEM) circuit model for the proposed filter in terms of a series connection of the multi-ports is presented and then used to predict the initial electrical and geometrical parameters. An experimental printed circuit prototype has been manufactured and evaluated to validate the design concept. The measured filter parameters correlate well with the simulation-derived ones and that increases the degree of freedom in the fabrication of microwave frequency selective components.
\end{abstract}

Keywords: bandstop filter; reentrant structure; multilayer implementation

\section{Introduction}

The microwave bandstop filters (BSFs) play an important role in the support of distortion reductions through the radio frequency (RF) and microwave communities [1]. For example, the BSFs requirements provide the highest possible transmission loss in the stop band in addition to a transmission increase away from the stop band. Many efforts have been pooled to realize various kinds of wideband BSFs [2-21]. Each such filter is a successful discovery in terms of insertion and return losses through the prescribed frequency band. The corresponding examples are as follows. First, Reference [3] contains Chapter 6 related to the various kinds of BSFs, including filters with narrow stop bands. These BSFs have stop-band bandwidths up to a few per cent, and equiripple or maximally flat characteristics in the pass-band. A strip-line filter and a wave guide one were successfully constructed. Second, in another study [7], two bandstop configurations were reported on. The first one accommodates even or odd characteristics and also asymmetric responses, although some negative or diagonal cross-couplings are needed. The second resembles the cul-de-sac configuration and needs no 
diagonal or negative couplings. The measured and simulation-derived results are in good agreement. Third, through the work in Reference [9], a general circuit configuration for cross-coupled wideband BSF was presented, where the cross-coupling between the input/output feed lines is realized by using a parallel-coupled line section. The distinct filtering properties of this type of transmission line filter are investigated. Fourth, the communication [13] deals with the BSF containing the parallel-coupled transmission line sections connected at both their ends. As a result, as much as five transmission zeros can be obtained. These zeros should be arranged to create a sharp rejection BSF. Fifth, the work in Reference [20] presents the dual-band microstrip BSF with nine transmission poles near the two stop bands. The centre frequencies of the BSF can be adjusted by changing even/odd-mode impedances of the coupled lines. However, as a rule, the used resonators resonate with the finite resistance of that and group-delay variation due to the steep phase change caused by the resonance. Moreover, the conventional BSFs contain the coupled-line sections arranged in various shapes [2-20].That is why, if a clearance in the two electromagnetically coupled strip (microstrip) transmission lines is unrealized, this then leads to significant discrepancies between the measured and simulation-derived results, including the impossibility of designed filter realization. This circumstance is an incentive for searching another alternative way of filter realization, when the two interacting transmission lines are initially uncoupled. Only at the final stage, both lines are arranged and mounted inside the third intermediate conductor with a floating potential that leads to the so-called "reentrant" structure [4].

Below, we propose for the first time a novel approach to design a reentrant structure with additional symmetrical reflection zeros at the lower and upper pass bands of the stop band, leading to the quasi-elliptic frequency response that improves the manufacturing and selectivity of the filter. Although the exact synthesis could almost certainly be performed, a relatively simple single-variable synthesis technique is employed, which is termed as "the synthesis by optimization" [8]. The corresponding transfer function plays here the role of a good starting point for optimization, ensuring that the optimization itself proceeds efficiently and rapidly. As a result, the TEM circuit model, full-wave simulation-derived results and measured ones are presented, and they show an acceptable agreement that paves the way for following applications of such filters in RF and microwave selective devices.

\section{Basic Structure}

The classical reentrant structure consists of three conductors $A, B, C$, and of the internal and external dielectric fillings with relative dielectric constants $\varepsilon_{r B}$ and $\varepsilon_{r N}$, respectively, as shown in Figure 1 [22]. These three conductors, each of physical length $l$, are arranged inside the pipe-like ground conductor $\mathrm{G}$ with a rectangular cross-section. The basic aspects of the design and realization of such a reentrant structure in solid and printed circuit board (PCB) implementations were proposed earlier [23-26]. It seems from these references that the device displayed in Figure 1 may be categorized as a symmetric four-port network with two symmetry axes.

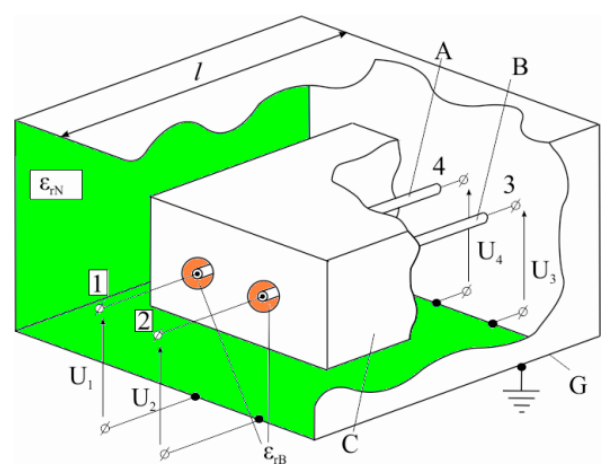

Figure 1. reentrant structure as a four-port network. A, B, C: conductors; G: ground conductor. 
If the metallic body $\mathrm{C}$ with a floating potential is implemented in other symmetrical (for example, trapezoidal) or asymmetrical (for example, shifted) shapes, then it is possible to realize the next generations of untraditional reentrant four-ports and corresponding reduced networks when one or more ports of a reentrant structure are not match-terminated. For example, if the "direct" and "coupled" ports of a classical backward-wave reentrant TEM directional coupler are open-circuited, the resulting reduced two-port network behaves as a bandpass filter [22].

Thus, the following sections are aimed at showing how the metallic body $\mathrm{C}$ modification leads to the creation of the quasi-elliptic bandstop filter. Generally speaking, through the conventional PCB implementation, both fillings play the role of supporting sheets with unequal dielectric constants $\left(\varepsilon_{r B} \neq \varepsilon_{r N}\right)$ and that satisfies a multilayer strip line realization. That is why it is necessary to describe the proposed two-port BSF using an adequate TEM circuit model which accounts for the reentrant nature and may be used for the search of electrical parameters with prescribed relative dielectric constants $\varepsilon_{r B}$ and $\varepsilon_{r N}$. The parameters predicted through the search by using nonlinear programming [27] can be taken as the appropriate initial quantities (in other words, as a proper starting point) in the full-wave simulation, as well as in a standard PCB realization.

\section{Filter Design}

\subsection{TEM Description of the Equivalent Filter Circuit}

To describe the proposed approach, including the key parameter optimization of the BSF, the corresponding decomposition was carried out. To begin the derivation of initial filter equations and corresponding optimal parameters, let us first consider the basic reentrant structure as a six-port device with the port designations shown in Figure 2a. According to Reference [28-30], this representation includes the symmetric impedance matrices $\left[Z_{B}\right]$ and $\left[Z_{N}\right]$ of two two-port networks. The first network is like the regular coaxial transmission line $1 \leftrightarrow 6$ (or $2 \leftrightarrow 5$ ) implemented inside the grounded body

$$
\left[Z^{B}\right]=\left[\begin{array}{ll}
Z_{11}^{B} & Z_{12}^{B} \\
Z_{12}^{B} & Z_{11}^{B}
\end{array}\right]
$$

whereas the second one is the regular coaxial line with the rectangular cross-section, in which the above-mentioned body itself plays the role of inner conductor without the previously inserted transmission lines $1 \leftrightarrow 6$ and $2 \leftrightarrow 5$.

$$
\left[Z^{N}\right]=\left[\begin{array}{ll}
Z_{11}^{N} & Z_{12}^{N} \\
Z_{12}^{N} & Z_{11}^{N}
\end{array}\right]
$$

Note that the ground conductor $\mathrm{G}$ is shown in Figure 2a and in the analogous following images, for simplicity, as the metallic plate rather than the metallic pipe with the rectangular cross-section. The corresponding equations for the matrix entireties were listed earlier [3,4,31].

Now, let us consider the four-port device containing the asymmetric shifted metallic body with the floating potential and port designations according to Figure $2 \mathrm{~b}$. After the decomposition of that as depicted in Figure 2c, we have the complex device consisting of the left and right four-port reentrant transmission lines with the corresponding metallic bodies designated by numbers " 1 " and " 3 ", and of the above-mentioned six-port circuit with the metallic body designated by number " 2 ". It is clear that this decomposition is fully equivalent to the four-port device with an asymmetric shifted body (Figure $2 b$ if the left node of body " 1 " and the right node of body " 3 " are open-circuited, as shown in Figure 2c, where the abbreviation "ocp" denotes the term "open-circuited port"). As a result, we have the so-called "shifted reentrant" four-port device, as briefly described in Reference [29], terminated by the transmission lines with the characteristic impedance $Z_{0}$ that is equal almost always to $50 \Omega$. 


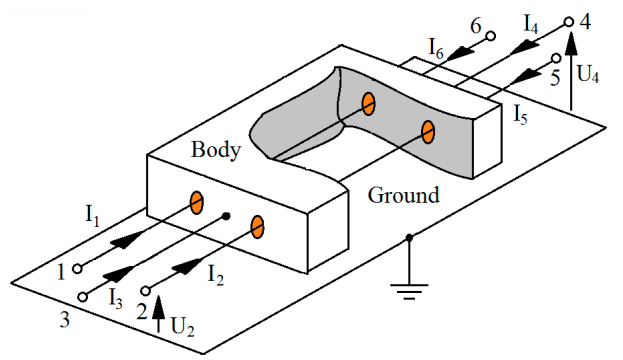

(a)

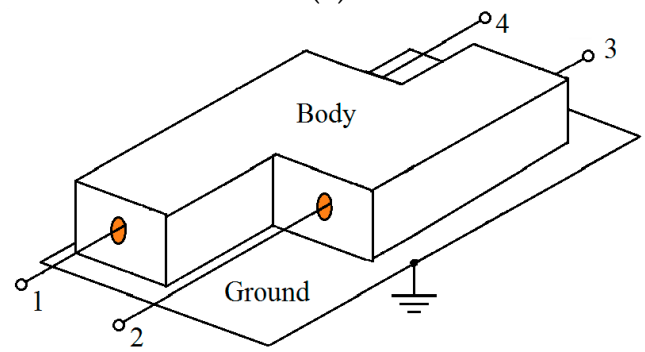

(b)

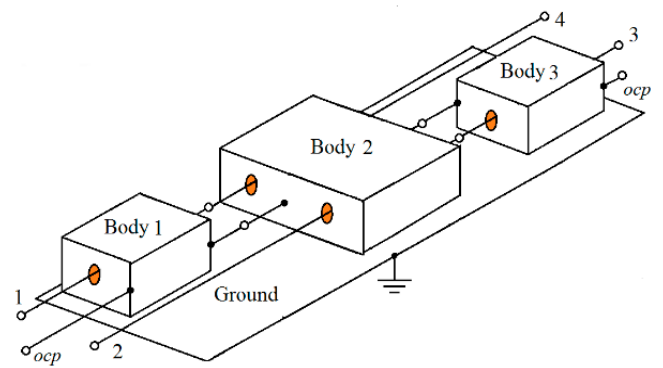

(c)

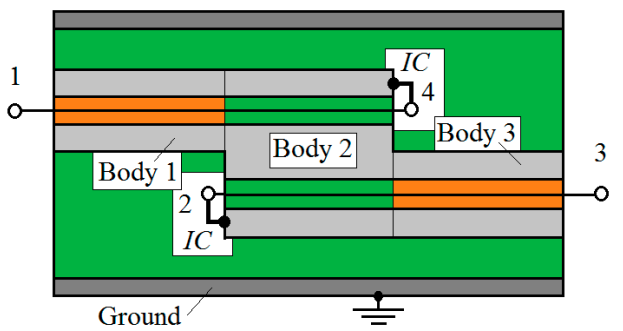

(d)

Figure 2. Proposed shifted reentrant device: (a) the six-port representation of the classical four-port reentrant structure, $(\mathbf{b})$ the shifted reentrant device, (c) the decomposition scheme, (d) the long-sectional view of the proposed quasi-elliptic bandstop filter(BSF) with various dielectric fillings (coaxial modification). ocp:open-circuited port.

After the scattering matrix

$$
[S]=\left[\begin{array}{llll}
S_{11} & S_{12} & S_{13} & S_{14} \\
S_{12} & S_{22} & S_{14} & S_{24} \\
S_{13} & S_{14} & S_{11} & S_{12} \\
S_{14} & S_{24} & S_{12} & S_{22}
\end{array}\right]
$$

that is calculated from the impedance matrices in Equations (1), (2), and other ones by using [32-35], as seen in Appendix A, one can find and analyze all the transfer functions of this device. The following performed detailed analysis has revealed that the shifted reentrant device itself shown in Figure $2 b$ does not have a practical purpose as the power divider because unacceptable 
values and frequency behaviors of the return losses $L_{1 R}=20 \log _{10}\left(\left|S_{11}\right|\right)$ and $L_{2 R}=20 \log _{10}\left(\left|S_{22}\right|\right)$ those are throughout the whole microwave frequency band.

However, for one of the reduced two-port circuits, an interesting expansion was found. Namely, if it is mandatory to use the direct interconnection (with the corresponding abbreviation "ic") of port 4 and the right border of body " 2 ", as well as of port 2 and the left border of that, the reduced two-port network with excited arms 1 and 3 behaves as the reciprocal bandstop filter with quasi-elliptic insertion loss response $L_{F}=20 \log _{10}\left(\left|S_{12}^{F}\right|\right)=20 \log _{10}\left(\left|S_{13}^{r e d u c}\right|\right)$ terminated by the transmission lines with characteristic impedance $Z_{B S}$. A long-sectional view of all the three bodies by the horizontal plane with the corresponding internal dielectric fillings (see below) is shown in Figure 2d. In other words, the scattering matrix $\left[S^{F}\right]$, as shown in Appendix A, of the proposed reciprocal BSF can be written as

$$
\left[S^{F}\right]=\left[\begin{array}{ll}
S_{11}^{F} & S_{12}^{F} \\
S_{12}^{F} & S_{11}^{F}
\end{array}\right]=\left[\begin{array}{ll}
S_{11}^{r e d u c} & S_{12}^{r e d u c} \\
S_{12}^{r e d u c} & S_{11}^{r e d u c}
\end{array}\right]
$$

where $S_{11}^{\text {reduc }}$ and $S_{13}^{\text {reduc }}$ denote the scattering parameters of the reduced two-port network as indicated above.

Both the scattering parameters of the proposed BSF versus frequency $f_{0 N}$ for $Z_{B L}=Z_{B S}=Z_{B R}$ $=20 \Omega$ and $Z_{N L}=Z_{N S}=Z_{N R}=60 \Omega$ with the same dielectric filling of the external regions of the proposed reentrant BSF, i.e., $\varepsilon_{r N L}=\varepsilon_{r N S}=\varepsilon_{r N R}=\varepsilon_{r N}$ are shown in Figure 3. Simultaneously, the internal dielectric fillings for $Z_{0}=50 \Omega$ are chosen as: $\varepsilon_{r B L}=\varepsilon_{r B R}=4 \varepsilon_{r N}$ (in Figure $2 \mathrm{~d}$ the corresponding areas are filled in orange colour) and $\varepsilon_{r B S}=\varepsilon_{r N}$ (these areas are filled in green colour), where $f_{0 N}=f_{0 N L}=f_{0 N S}=f_{0 N R}$ is the reference frequency of the device at which $\theta_{N L}=\theta_{N S}=\theta_{N R}=\pi / 2$. This result demonstrates only the behaviour of both transfer functions if the port 1 , as shown in Figure $2 \mathrm{~d}$, is the input one. Note that the above-mentioned selection of the internal fillings leads to the following coefficients through the supporting information:

$$
a_{B L}=\sqrt{\frac{\varepsilon_{r B L}}{\varepsilon_{r N L}}}=2, a_{B S}=\sqrt{\frac{\varepsilon_{r B S}}{\varepsilon_{r N S}}}=1, a_{B R}=\sqrt{\frac{\varepsilon_{r B R}}{\varepsilon_{r N R}}}=2 .
$$

Additionally, the use of the prescribed requirements $Z_{B L}=Z_{B S}=Z_{B R}$ and $Z_{N L}=Z_{N S}=Z_{N R}$ may be greatly conductive to simplify the following multilayer implementation (see Section 4). Thus, the proposed BSF has the infinite numbers of the stop bands centred about the odd multiplies of frequency $f^{*}=f_{0 N} / 3$.

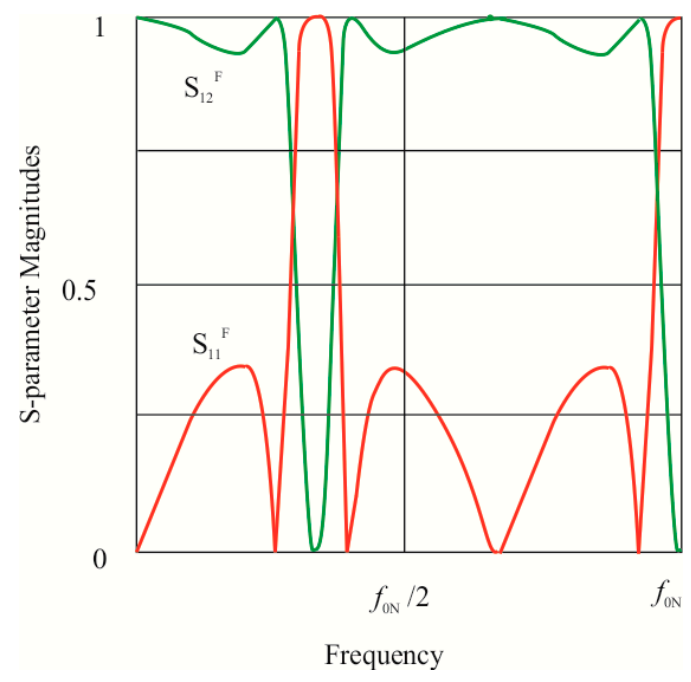

(a)

Figure 3. Cont. 


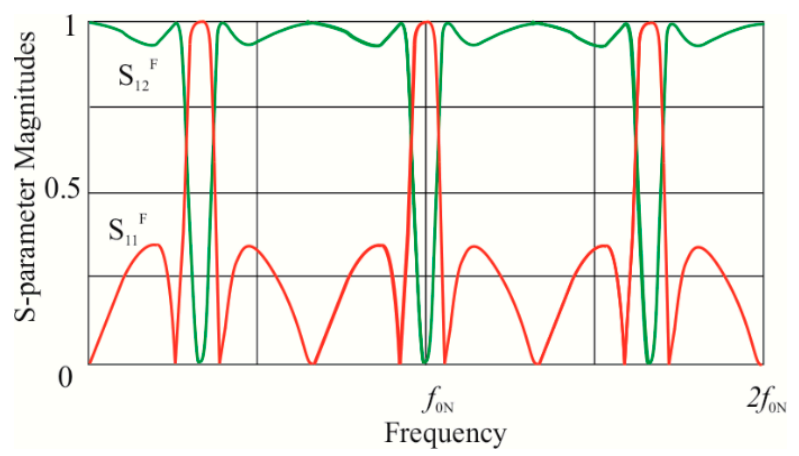

(b)

Figure 3. S-parameter magnitudes of the proposed bandstop filter on the base of its TEM model: (a) for the frequency band from 0 up to the reference frequency, (b) through band 0 -double reference frequency.

\subsection{Full-Wave Simulations}

To confirm an adequacy of the described TEM algorithm, the proposed BSF was simulated through the three-dimensional (3D) electromagnetic (EM) solver [36] with the above-mentioned characteristic impedances (Section 3.1) for the vacuum filling of the regions with relative permittivity $\varepsilon_{r N}$, i.e., $\varepsilon_{r N}=1$, as shown in green in Figure $2 \mathrm{~d}$. The 3D views of the filter using the coaxial lines at large and fine scales are shown in Fig. 4. Namely, the general view of the coaxial model with port locations is given in Figure $4 \mathrm{a}$. The perspective view of port 1 is shown in Figure $4 \mathrm{~b}$ on the fine scale. The inner entirety of the left filter butt-end with partly deleted metallic cylinders and dielectric fillings including the internal medium with relative permittivity $\varepsilon_{r B L}=4 \varepsilon_{r N}=4$ (orange colouring), is illustrated in Figure $4 \mathrm{c}$ on the fine scale. The perspective view of the filter entireties with significantly deleted metallic cylinders and dielectric pipes is shown in Figure $4 \mathrm{~d}$, also on the fine scale. The region near the virtual border between bodies " 1 " and " 2 " is given in Figure $4 \mathrm{e}$ on the very fine scale, whereas the analogous region near the virtual border between bodies " 2 " and " 3 " is shown in Figure $4 \mathrm{f}$ on the same scale. The border vertical plane, which is used to remove the cylinders and pipes, is placed at the point of the direct interconnection between port 4 and the right virtual border of body " 2 " inside it. The general view of the filter in the other foreshortening using the vertical rotation by $90^{\circ}$, with respect to the image of Figure 4a, is shown in Figure $4 \mathrm{~g}$ that is used for a better understanding of the second port location, as shown in Figure 4h, fine scale.

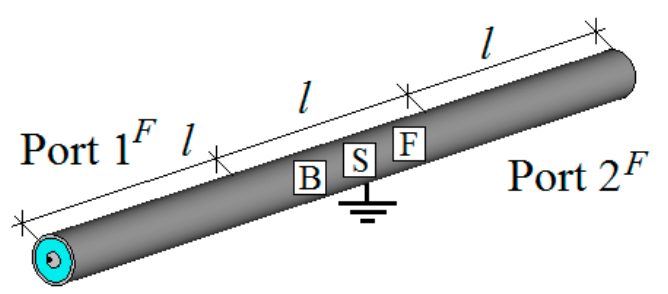

(a)

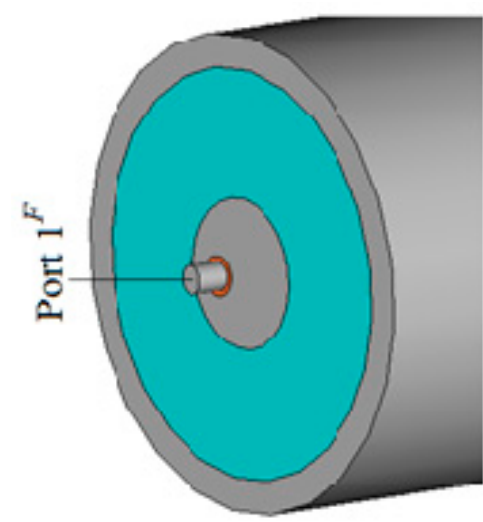

(b)

Figure 4. Cont. 


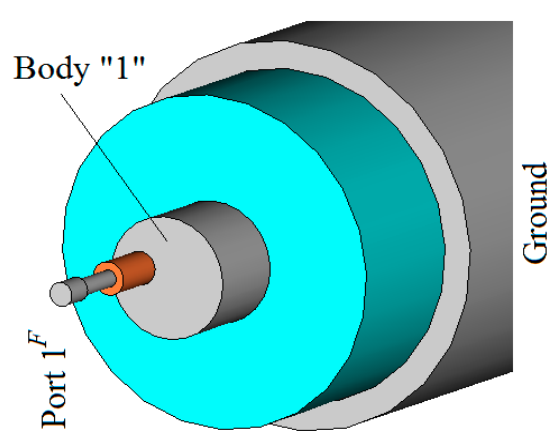

(c)

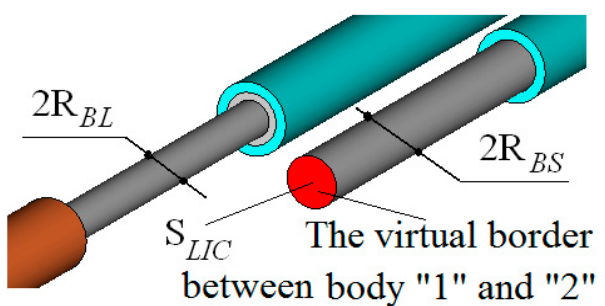

(e)

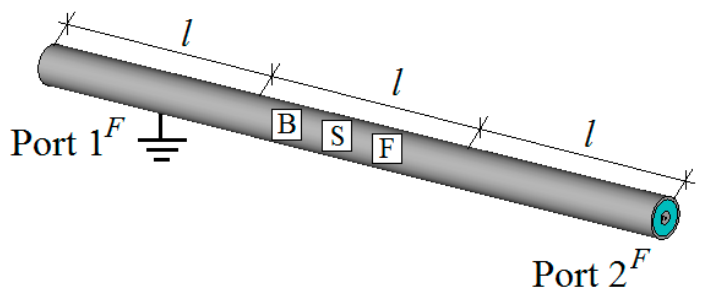

(g)

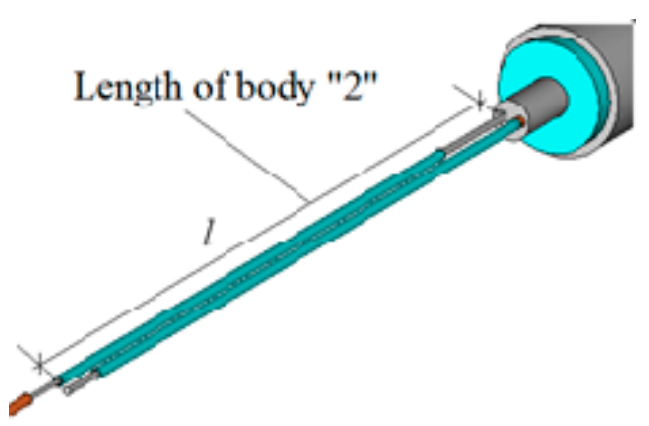

(d)

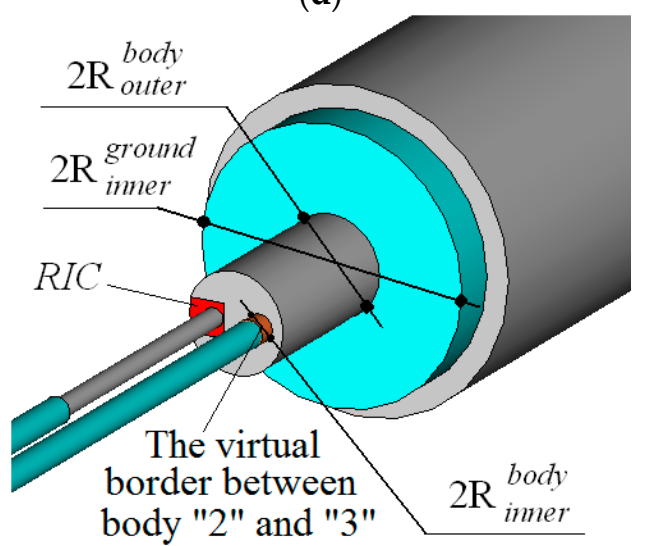

(f)

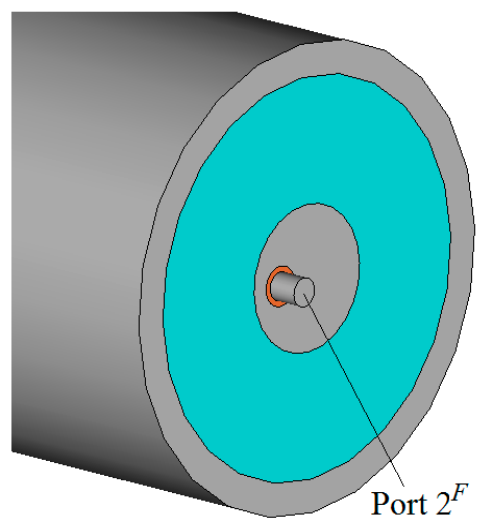

(h)

Figure 4. Proposed coaxial bandstop filter: (a) general 3D view, (b) 3D view of the left butt-end (fine scale), (c) the latest 3D view on the very fine scale, (d) 3D view of the entirety of body " 2 " (fine scale), (e) 3D view of the region near the left virtual border of body "2" (very fine scale), (f) 3D view of the region near the right virtual border of body " 2 " (the same scale), (g) general 3D view in the other foreshortening, (h) 3D view of the right butt-end of the filter with the second port location (fine scale). LIC:left interconnection; RIC: right interconnection.

The corresponding radii of the above-mentioned coaxial model as shown in Figure 4 are as follows:

- the first halves of the solid metallic cylinders inside bodies " 1 " and " 3 " are surrounded by the orange medium with relative dielectric constant $\varepsilon_{r B L}=\varepsilon_{r B R}=4: R_{B L}=R_{B R}=0.308 \mathrm{~mm}$;

- the second halves of the solid metallic cylinders inside body " 2 " are surrounded by the green vacuum filling: $R_{B S}=0.43 \mathrm{~mm}$;

- $\quad$ the inner radius of both solitary holes inside the solid cylinder with a floating potential that plays the role of all three bodies " 1 ", " 2 ", and " 3 " correspondingly connected in echelon: 
$R_{\text {inner }}^{\text {body }}=0.6 \mathrm{~mm}$, i.e., for $\varepsilon_{r B L}=\varepsilon_{r B R}=4$ (orange medium) and $\varepsilon_{r B S}=1$ (green one) we have $Z_{B L}=Z_{B R}=30 \log \left(R_{\text {inner }}^{\text {body }} / R_{B L}\right)=Z_{N S}=60 \log \left(R_{\text {inner }}^{\text {body }} / R_{B S}\right)=20 \Omega$;

- $\quad$ the outer radius of the cylinder with a floating potential: $R_{\text {inner }}^{\text {body }}=2.205 \mathrm{~mm}$;

- the inner radius of a grounded cylinder related to all the three bodies: $R_{\text {inner }}^{\text {ground }}=6 \mathrm{~mm}$, i.e., for $\varepsilon_{r N L}=\varepsilon_{r N S}=\varepsilon_{r N R}=1$ (green medium) we have $Z_{N L}=Z_{N S}=Z_{N R}=$ $60 \log \left(R_{\text {inner }}^{\text {ground }} / R_{\text {inner }}^{\text {body }}\right)=60 \Omega$;

- $\quad$ the outer radius of the grounded cylinder is chosen as $7 \mathrm{~mm}$.

The length of each of the three bodies, as shown in Figures $2 \mathrm{~d}$ and $4 \mathrm{a}, \mathrm{g}$ : $l=89 \mathrm{~mm}$ $\left(f_{0 N}=0.843 \mathrm{GHz}\right)$. Note that a butt-end of the cylinder with cross-section $S_{\text {LIC }}$ (red colouring) forms at the bottom of the corresponding solitary hole (not shown in Figure 4), the left interconnection (the corresponding abbreviation in the last index is "LIC"), between the left border of body " 2 " and port 2 of the four-port network, as shown in Figure 2c, whereas the region designated by the abbreviation "RIC" (also in red colouring) relates to the interconnection between the right border of body " 2 " and port 4 of the same four-port circuit.

The simulated results, as shown in Figure 5, and their comparison with the curves shown in Figure 3 demonstrate the validity of the presented TEM algorithm and its usefulness for the prediction of optimal filter electrical parameters.

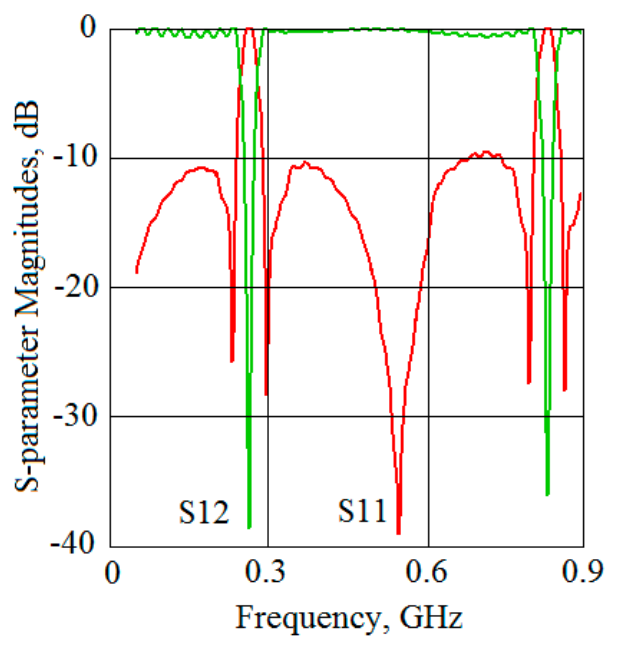

Figure 5. S-parameter magnitudes of the coaxial bandstop filter model.

\section{Experimental Results}

In the above section, we have used the TEM-mode algorithm to predict the initial electrical parameters of the filter. Below, the analogous results are applied for the determination of the corresponding reentrant printed-circuit dimensions. Let us select the central frequency of the filter set equal to $600 \mathrm{MHz}$ with the corresponding electrical lengths $\theta_{N L}=\theta_{N S}=\theta_{N R}=\pi / 2$, that is: $f_{0 N L}=f_{0 N S}=f_{O N R}=f_{0 N}=600 \mathrm{MHz}$. Additionally, the level of the ripples of $\left|S_{11}^{F}\right|$ in the pass band is chosen as $(-15) \mathrm{dB}$, and that leads to the following Fortran-derived impedances after the use of the conjugate gradient optimization [27]: $Z_{B L}=Z_{B S}=Z_{B R}=20 \Omega$ and $Z_{N L}=Z_{N R}=Z_{N S}=47 \Omega$. In other words, the return loss response $L_{R}=20 \log _{10}\left(\left|S_{11}^{F}\right|\right)$ (see Equation (4)) plays the role of the goal function of two independent variables $\left(Z_{B L}, Z_{N L}\right)$ through the nonlinear optimization process by using the conjugate gradient algorithm [27] converging rapidly towards the minimum/maximum point. If the maximum of the goal function (it has the response like the red curve in Figure 5) is equal to $(-15) \mathrm{dB}$ with the deviation $\pm 0.05 \mathrm{~dB}$, then the optimization process is stopped. As a result, we have the corresponding impedances 20 and $47 \Omega$. 
The filter was manufactured in the conventional four-layer strip line technique [23-25] on the commercially available Russian substrates as the supporting sheets. The authors understand that, for the prototype description, it would be more preferable to use a certain Russian book systematizing the data on the Russian materials, technologies, and manufacturing firms of these materials. A very good example of such book is Reference [5]. However, it has turned out that the data on the Russian materials and technologies are dispersed over several books. Thus, several contributions in Russian are included in the reference list.

As a result, the Russian FF-4 substrate [37] without copper clothes was chosen for the manufacturing of outer sheets because of its low loss tangent $(\tan \delta=0.0003)$ and a small dielectric constant variation $\left(\varepsilon_{r N L}=\varepsilon_{r N S}=\varepsilon_{r N R}=2.0 \pm 0.1\right)$. Further, it is expedient to emphasize that the use of various dielectric media as the internal fillings of bodies " 1 " (or "3") and "2" (see Section 3.2) is almost always unacceptable in manufacturing. On the contrary, it is more expedient to use the variant when the lines inside bodies " 1 " and " 3 " have a double geometrical length and are meandered along the longitudinal axis of the filter between the virtual borders of these bodies inside them. This offers the possibility to use the analogous dielectric nomenclature as the internal filling of the filter. Thus, in the following PCB implementation, only the relative dielectric constant 2.0 is used. That is why the thin central boards $G_{1}$ and $G_{2}$ of the filter, as shown in Figure 6a, were realized on the base of the Russian thin film material F4MBSF $\left(\varepsilon_{r B L}=\varepsilon_{r B S}=\varepsilon_{r B R}=2.0 Z_{12}^{B L}=Z_{B L} \beta_{B L} 0.15\right) 0.12 \mathrm{~mm}$ thick and, initially, $\mathrm{Cu}$-clad on both sides [38]. The copper foil thickness $t_{F}$ is equal to $0.02 \mathrm{~mm}$. That is, the central thin boards $G_{1}$ and $G_{2}$ with the corresponding thickness $S_{1}=0.12 \mathrm{~mm}$ (F4MBSF), as shown in Figure 6a, were placed between the outer sheets $G_{3}$ and $G_{4}$ with their thickness $S_{2}$, as shown in Figure $6 \mathrm{~b}$. Since all the lines are initially uncoupled, the formulas from Reference [5] were used for calculations:

- $\quad$ the impedances $Z_{N L}=Z_{N S}=Z_{N R}=47 \Omega$ are realized as the relative thin metallic bar of width $W_{N}$ and height $t_{N}=2 S_{1}+3 t_{F}=0.3 \mathrm{~mm}$ placed symmetrically between the ground planes with cross-sectional distance $b_{N}=2 S_{2}+t_{N}$, as shown in Figure 6b;

- $\quad$ the impedances $Z_{B L}=Z_{B S}=Z_{B R}=20 \Omega$ are realized as the regular strip lines of thickness $t_{F}$ and width $W_{B}$ placed symmetrically between the ground planes with the common distance $b_{B}=2 S_{1}+t_{F}=0.26 \mathrm{~mm}$.

According to our understanding, the dimension search is a fortuitous process based on trial-and-error procedures. Starting from the approach related to the previously manufactured reentrant devices [39-41], it is possible to determine the following dimensions which are summarized below (in millimetres) and shown in Figure 6: $S_{2}=5$ (the standard thickness of FF-4 substrate [37]); $S_{3}=2 ; S_{4}=0.2 ; S_{5}=17.125 ; l_{1}=5 ; l_{2}=11.75 ; l_{3}=12.5 ; l_{4}=24.25 ; l_{5}=25 ; l_{0 N}=30 ; S_{B}=1.75 ;$ $S_{N}=15.375 ; W_{0}=9.1 ; W_{B}=0.75 ; W_{N}=9.25$.

The whole assemblage of the proposed reentrant bandstop filter includes the implementation of six metallized holes being $0.5 \mathrm{~mm}$ in diameter, as shown in Figure 6a (the corresponding abbreviation is "MH"), placed at the ends of the entire lines lying inside body " 2 ", as well as at the corners of the rectangular conductor with dimension $W_{N} \times 3 l_{0 N}$. The latest four holes ensure the required surface potential conditions on both outer sides of the printed conductor $W_{N} \times 3 l_{0 N}$ which plays the role of all the bodies " 1 ", " 2 ", and " 3 " connected in an echelon.

A photograph of the central board $G_{1}$ is shown in Figure 7. The common dimensions, excluding two SubMiniature version A (SMA) connectors and the metallic walls of the filter mounting, are $104 \times 40 \mathrm{~mm}^{2}$. The measurements were performed with the use of a DS 7710A Network Analyser "Deviser" (the characteristic impedance $Z_{0}=50 \Omega$ ) operating over the frequency range $0.3-1.3 \mathrm{GHz}$, which utilizes the short-open-load-thru (SOLT) calibration. The inner conductors of SMA connectors were soldered to the printed fragments, of which the dimensions are $W_{0} \times l_{1}$, as shown in Figure 6a. After the corresponding reciprocal assemblage of all the boards inside a proper metallic mounting, we have the proposed four-layer reentrant BSF. As seen in Figure 8, the simulated and measured responses are in a good agreement. A slight discrepancy in the frequency shifting may primarily be caused by 
the temperature variations of $\varepsilon_{r B}$ and $\varepsilon_{r N}$, changes in dielectric constants from lot to lot, substrate thickness variations, and, finally, by the unexpected fabrication tolerances and parasitic effects of the SMA connectors. The measured insertion loss in the upper pass band, including the material losses and two SMA connectors, was better than $1.3 \mathrm{~dB}$.

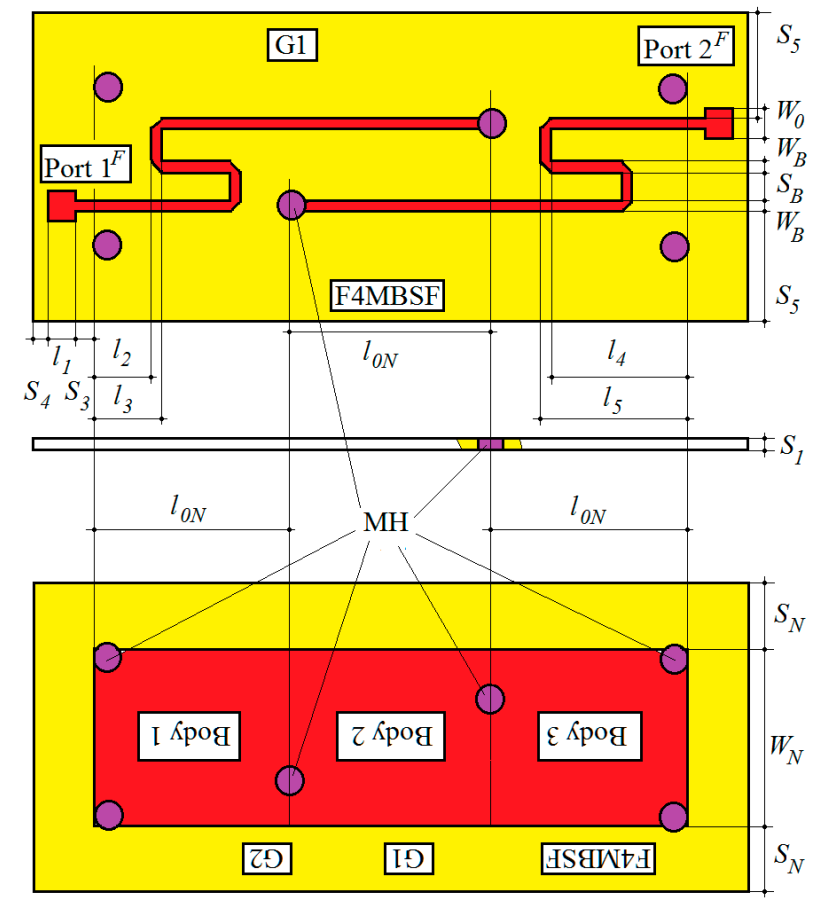

(a)

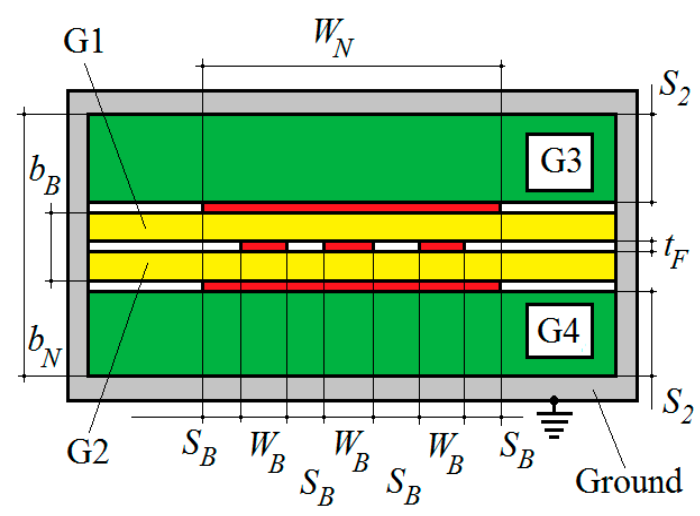

(b)

Figure 6. Architecture of the proposed four-layer reentrant bandstop filter: (a) face and bottom

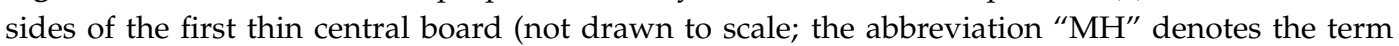
"metallized hole"); note that the face side the second central board is realized without copper clothes, (b) cross-sectional view at the centre of body " 1 " after the corresponding reciprocal assemblage (not drawn to scale). F4MBSF:Russian thin film material. 


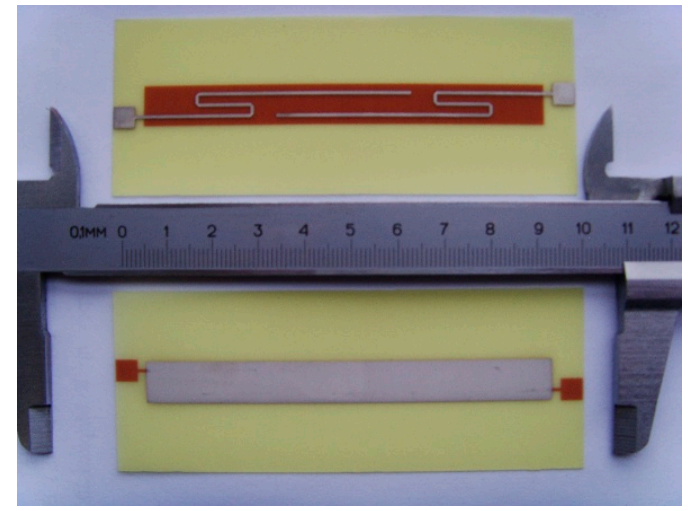

Figure 7. A photograph of the first central board without the metallized holes.

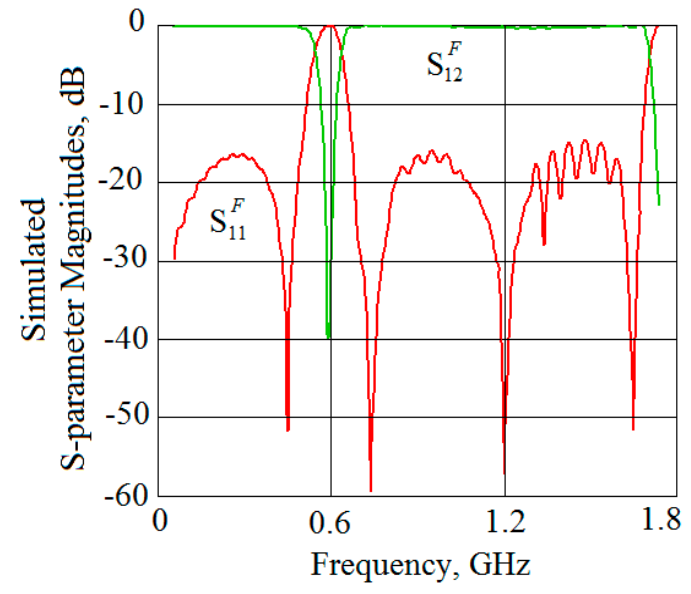

(a)

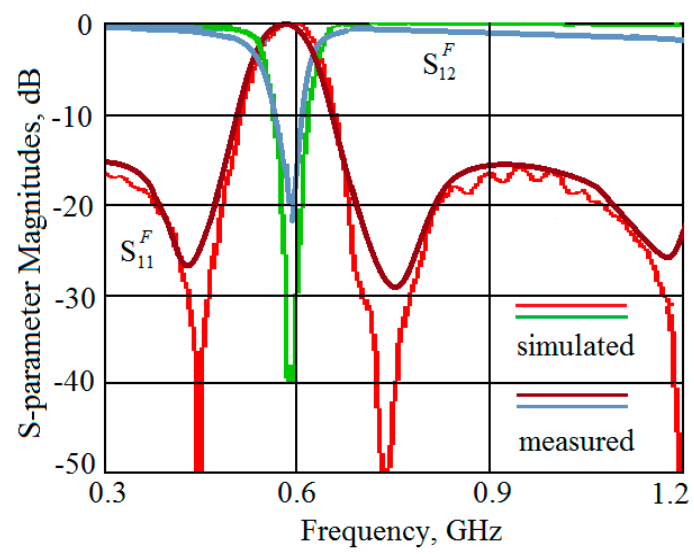

(b)

Figure 8. Measured and simulated S-parameters of the proposed bandstop filter: (a) the simulation-derived results in the frequency range $0.1-1.8 \mathrm{GHz},(\mathbf{b})$ these results, as well as the experimental ones, are through the whole frequency band of the DS 7710A Network Analyser "Deviser".

\section{Discussion}

Thus, the proposed approach offers the possibility of creation of the various filtering devices on the base of untraditional reentrant structures comprising the metallic bodies with floating potential. The specific feature of these untraditional structures is the various forms of the bodies, including asymmetrical, shifted, or symmetrical ones, realized on the base of the uniform transmission lines. 
Moreover, these bodies may be realized on the base of the nonuniform tapered transmission lines. Additionally, such tapered lines may be also used as the internal transmission channels inside the bodies with floating potential.

Generally speaking, an imagination is irrepressible. For example, the situation can be considered, when the body with floating potential plays the role of a low-pass filter, whereas the internal transmission channel acts as the high-pass one or vice versa, and so on. Additionally, the reduced two-port networks containing the various combinations of short-, open-, or interconnected circuits on the unenergized nodes can be designed. Thus, the proposed approach paves the way to realize the filters, power splitters, phasers (phase shifters), power dividers with various division ratios and additional degrees of freedom. Moreover, it is evidence to analyse the surface distribution of an electric current carried by the external surface of the body with floating potential. If the ground pipe-like conductor is omitted, then a reduced reentrant device may be used as the untraditional radiating element capable of performing the next generation of antenna systems.

\section{Conclusions}

A novel bandstop filter was developed on a untraditional reentrant configuration base. The proper selection of the impedances and relative permittivities of their transmission lines was performed with the use of the "synthesis by optimization" approach. The measured results are in reasonable agreement with the TEM and full-wave predicted data and suggest that the proposed filter with initially uncoupled strip lines has attractive performances and acceptable dimensions. Due to the asymmetric metallic body with the floating potential, the proposed reentrant filter, as a basic building block, is expected to be used in various types of frequency selective devices that pave the way for a variety of applications, including LTCC and SIW technologies.

Author Contributions: Methodology, V.V.A.; software, validation, and formal analysis, A.P.G. and V.A.K.; investigation, N.V.T.

Funding: This study was supported by the Ministry of Science and Higher Education of the Russian Federation (8.6847.2017/BCh) and by project C-13, the research issue: "Design of dual-band dipole antennas with an essential working frequency diversity" with the financial support of the Novosibirsk State Technical University internal grant for young scientists. Also, this work was supported by the Russian Foundation for Basic Research (16-52-48010, 17-52-53031).

Conflicts of Interest: The authors declare no conflict of interest.

\section{Appendix A}

To derive the overall scattering matrix $\left[S^{F}\right]$ in Equation (4) of the proposed BSF, as shown in Figure $2 \mathrm{~d}$, we start from the scattering matrix $\left[S^{6}\right]$ of the six-port device realized on the base of body "2", as shown in Figure 2c:

$$
\left[S^{6}\right]=\left[\begin{array}{llllll}
S_{11}^{6} & S_{12}^{6} & S_{13}^{6} & S_{14}^{6} & S_{15}^{6} & S_{16}^{6} \\
S_{21}^{6} & S_{22}^{6} & S_{23}^{6} & S_{24}^{6} & S_{25}^{6} & S_{26}^{6} \\
S_{31}^{6} & S_{32}^{6} & S_{33}^{6} & S_{34}^{6} & S_{35}^{6} & S_{36}^{6} \\
S_{41}^{6} & S_{42}^{6} & S_{43}^{6} & S_{44}^{6} & S_{45}^{6} & S_{46}^{6} \\
S_{51}^{6} & S_{52}^{6} & S_{53}^{6} & S_{54}^{6} & S_{55}^{6} & S_{56}^{6} \\
S_{61}^{6} & S_{62}^{6} & S_{63}^{6} & S_{64}^{6} & S_{65}^{6} & S_{66}^{6}
\end{array}\right]
$$

This matrix was formed by the conversion between the impedance matrix $\left[Z^{6}\right]$ of the six-port and scattering one $\left[S^{6}\right]$, as described in Reference [32]. Applying the technique proposed in Reference [30], one can write the following relations for matrix $\left[Z^{6}\right]$ : 


$$
\left[Z^{6}\right]=\left[\begin{array}{cccccc}
Z_{11}^{B S}+Z_{11}^{N S} & Z_{11}^{N S} & Z_{11}^{N S} & Z_{12}^{N S} & Z_{12}^{N S} & Z_{12}^{B S}+Z_{12}^{N S} \\
Z_{11}^{N S} & Z_{11}^{B S}+Z_{11}^{N S} & Z_{11}^{N S} & Z_{12}^{N S} & Z_{12}^{B S}+Z_{12}^{N S} & Z_{12}^{N S} \\
Z_{11}^{N S} & Z_{11}^{N S} & Z_{11}^{N S} & Z_{12}^{N S} & Z_{12}^{N S} & Z_{12}^{N S} \\
Z_{12}^{N S} & Z_{12}^{N S} & Z_{12}^{N S} & Z_{11}^{N S} & Z_{11}^{N S} & Z_{11}^{N S} \\
Z_{12}^{N S} & Z_{12}^{B S}+Z_{12}^{N S} & Z_{12}^{N S} & Z_{11}^{N S} & Z_{11}^{B S}+Z_{11}^{N S} & Z_{11}^{N S} \\
Z_{12}^{B S}+Z_{12}^{N S} & Z_{12}^{N S} & Z_{12}^{N S} & Z_{11}^{N S} & Z_{11}^{N S} & Z_{11}^{B S}+Z_{11}^{N S}
\end{array}\right]
$$

where

$$
\begin{gathered}
Z_{11}^{B S}=Z_{B S} \alpha_{B S}, Z_{12}^{B S}=Z_{B S} \beta_{B S}, Z_{11}^{N S}=Z_{N S} \alpha_{N S}, Z_{12}^{N S}=Z_{N S} \beta_{N S}, \\
\alpha_{B S}=-j \cot \theta_{B S}, \beta_{B S}=-j \csc \theta_{B S}, \alpha_{N S}=-j \cot \theta_{N S}, \beta_{N S}=-j \csc \theta_{N S}, \\
\theta_{B S}=a_{B S}(\pi / 2)\left(f / f_{0 N S}\right), a_{B S}=\sqrt{\varepsilon_{r B S} / \varepsilon_{r N S}}, \theta_{N S}=(\pi / 2)\left(f / f_{0 N S}\right) .
\end{gathered}
$$

The latest equations comprise the characteristic impedances $Z_{B S}$ and $Z_{N S}$ of the transmission line 1 " $\leftrightarrow 6^{\prime \prime}$ [30] inside the dielectric filling $\varepsilon_{r B S}$ and of the body " 2 " itself placed inside the dielectric filling $\varepsilon_{r N S}$, respectively, whereas $f_{0 N S}$ is the reference frequency of body " 2 ", as shown in Figure 2c, $\mathrm{d}$ in the main text, at which $\theta_{N S}=\pi / 2$.

The following stage of the analysis includes the description of the reduction procedure in order to realize the interconnection between port 2, as shown in Figure A1a, and the left edge of body "2", as shown in Figure 2a in the main text. This procedure is illustrated by Figure A1a where the corresponding ports of the equivalent five-port device are designated by the lower case letters " $a$ ", "b", "c", "d", and "e". Applying the technique described in Reference [33], one can write:

$$
\left[S^{5}\right]=\left[\begin{array}{ccccc}
S_{a a}^{5} & S_{a b}^{5} & S_{a c}^{5} & S_{a d}^{5} & S_{a e}^{5} \\
S_{b a}^{5} & S_{b b}^{5} & S_{b c}^{5} & S_{b d}^{5} & S_{b e}^{5} \\
S_{c a}^{5} & S_{c b}^{5} & S_{c c}^{5} & S_{c d}^{5} & S_{c e}^{5} \\
S_{d a}^{5} & S_{d b}^{5} & S_{d c}^{5} & S_{d d}^{5} & S_{d e}^{5} \\
S_{e a}^{5} & S_{e b}^{5} & S_{e c}^{5} & S_{e d}^{5} & S_{e e}^{5}
\end{array}\right]=\left[S_{P P}\right]+\left[S_{P C}\right]\left([F]-\left[S_{C C}\right]\right)^{-1}\left[S_{C P}\right]
$$

where

$$
\begin{gathered}
{\left[S_{P P}\right]=\left[\begin{array}{ccccc}
S_{11}^{6} & 0 & S_{15}^{6} & S_{14}^{6} & S_{16}^{6} \\
0 & S_{11}^{T L} & 0 & 0 & 0 \\
S_{51}^{6} & 0 & S_{55}^{6} & S_{54}^{6} & S_{56}^{6} \\
S_{41}^{6} & 0 & S_{45}^{6} & S_{44}^{6} & S_{46}^{6} \\
S_{61}^{6} & 0 & S_{65}^{6} & S_{64}^{6} & S_{66}^{6}
\end{array}\right],\left[S_{P C}\right]=\left[\begin{array}{cccc}
0 & S_{13}^{6} & 0 & S_{12}^{6} \\
S_{12}^{T L} & 0 & S_{13}^{T L} & 0 \\
0 & S_{53}^{6} & 0 & S_{52}^{6} \\
0 & S_{43}^{6} & 0 & S_{42}^{6} \\
0 & S_{63}^{6} & 0 & S_{62}^{6}
\end{array}\right], \quad\left[\begin{array}{ccccc}
0 & & \\
{\left[S_{C P}\right]} & S_{21}^{T L} & 0 & 0 & 0 \\
S_{31}^{6} & 0 & S_{35}^{6} & S_{34}^{6} & S_{36}^{6} \\
0 & S_{31}^{T L} & 0 & 0 & 0 \\
S_{21}^{6} & 0 & S_{25}^{6} & S_{24}^{6} & S_{26}^{6}
\end{array}\right],\left[S_{C C}\right]=\left[\begin{array}{ccccc}
S_{22}^{T L} & 0 & S_{23}^{T L} & 0 \\
0 & S_{33}^{6} & 0 & S_{32}^{6} \\
S_{32}^{T L} & 0 & S_{33}^{T L} & 0 \\
0 & S_{23}^{6} & 0 & S_{22}^{6}
\end{array}\right],[F]=\left[\begin{array}{llll}
0 & 0 & 0 \\
1 & 0 & 0 & 0 \\
0 & 0 & 0 & 1 \\
0 & 0 & 1 & 0
\end{array}\right] .}
\end{gathered}
$$

The latest matrices comprise the elements of the scattering matrix $\left[S^{T L}\right]$ related to the three-port network that models the above-mentioned interconnection at the left side of body " 2 ". This three-port is shown in Figure A1a including the port designations by the numbers $1^{L}, 2^{L}$, and $3^{L}$ with the above indices by the capital letter " $L$ " which denotes the relation to the left side of body " 2 ". According to Reference [34], matrix $\left[S^{T L}\right]$ may be written as

$$
\left[S^{T L}\right]=\left[\begin{array}{lll}
S_{11}^{T L} & S_{12}^{T L} & S_{13}^{T L} \\
S_{21}^{T L} & S_{22}^{T L} & S_{23}^{T L} \\
S_{31}^{T L} & S_{32}^{T L} & S_{33}^{T L}
\end{array}\right]=\left[\begin{array}{ccc}
-\frac{1}{3} & \frac{2}{3} & \frac{2}{3} \\
\frac{2}{3} & -\frac{1}{3} & \frac{2}{3} \\
\frac{2}{3} & \frac{2}{3} & -\frac{1}{3}
\end{array}\right]
$$

To continue the reduction procedure from the six-port network into the four-port one, it is necessary to realize the interconnection between port 6, as shown in Figure A1a, and the right edge of 
body " 2 ", as shown in Figure 2c. This continuation is illustrated in Figure A1b, where the corresponding ports of the equivalent four-port device are designated by the capital letters A, B, C, and D. Applying the technique described in Reference [33], one can write

$$
\left[S^{4}\right]=\left[\begin{array}{llll}
S_{A A}^{4} & S_{A B}^{4} & S_{A C}^{4} & S_{A D}^{4} \\
S_{B A}^{4} & S_{B B}^{4} & S_{B C}^{4} & S_{B D}^{4} \\
S_{C A}^{4} & S_{C B}^{4} & S_{C C}^{4} & S_{C D}^{4} \\
S_{D A}^{4} & S_{D B}^{4} & S_{D C}^{4} & S_{D D}^{4}
\end{array}\right]=\left[S_{P P}^{\prime}\right]+\left[S_{P C}^{\prime}\right]\left(\left[F^{\prime}\right]-\left[S^{\prime} C C\right]\right)^{-1}\left[S_{C P}^{\prime}\right]
$$

where

$$
\begin{gathered}
{\left[S_{P P}^{\prime}\right]=\left[\begin{array}{cccc}
S_{a a}^{5} & S_{a b}^{5} & S_{a c}^{5} & 0 \\
S_{b a}^{5} & S_{b b}^{5} & S_{b c}^{5} & 0 \\
S_{c a}^{5} & S_{c b}^{5} & S_{c c}^{5} & 0 \\
0 & 0 & 0 & S_{11}^{T R}
\end{array}\right],\left[S_{P C}^{\prime}\right]=\left[\begin{array}{cccc}
S_{a e}^{5} & 0 & S_{a d}^{5} & 0 \\
S_{b e}^{5} & 0 & S_{b d}^{5} & 0 \\
S_{c e}^{5} & 0 & S_{c d}^{5} & 0 \\
0 & S_{12}^{T R} & 0 & S_{13}^{T R}
\end{array}\right],} \\
{\left[S_{C P}^{\prime}\right]=\left[\begin{array}{cccc}
S_{e a}^{5} & S_{e b}^{5} & S_{e c}^{5} & 0 \\
0 & 0 & 0 & S_{21}^{T R} \\
S_{d a}^{5} & S_{d b}^{5} & S_{d c}^{5} & 0 \\
0 & 0 & 0 & S_{31}^{T R}
\end{array}\right],\left[S_{C C}^{\prime}\right]=\left[\begin{array}{cccc}
S_{e e}^{5} & 0 & S_{e d}^{5} & 0 \\
0 & S_{22}^{T R} & 0 & S_{23}^{T R} \\
S_{d e}^{5} & 0 & S_{d d}^{5} & 0 \\
0 & S_{32}^{T R} & 0 & S_{33}^{T R}
\end{array}\right],\left[F^{\prime}\right]=[F]}
\end{gathered}
$$

The latest matrices contain the elements of the scattering matrix $\left[S^{T R}\right]$ related to the three-port device that models, on the analogy with Figure A1a, the interconnection at the right side of body " 2 ". This three-port network is illustrated in Figure A1b including the port designations by numbers $1^{R}, 2^{R}$, and $3^{R}$ with the corresponding indices by the capital letter " $\mathrm{R}^{\text {" }}$ that denotes the relation with the right side of body " 2 ". It is clear that matrices $\left[S^{T R}\right]$ and $\left[S^{T L}\right]$ are equal for the prescribed port designations, as shown in Figure A1:

$$
\left[S^{T R}\right]=\left[\begin{array}{lll}
S_{11}^{T R} & S_{12}^{T R} & S_{13}^{T R} \\
S_{21}^{T R} & S_{22}^{T R} & S_{23}^{T R} \\
S_{31}^{T R} & S_{32}^{T R} & S_{33}^{T R}
\end{array}\right]=\left[\begin{array}{ccc}
-\frac{1}{3} & \frac{2}{3} & \frac{2}{3} \\
\frac{2}{3} & -\frac{1}{3} & \frac{2}{3} \\
\frac{2}{3} & \frac{2}{3} & -\frac{1}{3}
\end{array}\right]
$$

The following stage of the analysis includes the consideration of the left four-port reentrant coaxial section realized on the base of body " 1 " and placed above the ground, as shown in Figure 2c. Through this stage it is mandatory to use the open-circuited regime at the left body port $2^{R L}$, as shown in Figure A2a. According to References $[30,34,35]$, the scattering matrix $\left[S^{R L}\right]$ of the three-port device reduced by such the regime, with arm numbering $1^{R L}, 3^{R L}$, and $4^{R L}$, may be written as (port $2^{R L}$ is open-circuited, in other words, it is omitted):

$$
\left[S^{R L}\right]=\left[\begin{array}{lll}
S_{11}^{R L} & S_{13}^{R L} & S_{14}^{R L} \\
S_{31}^{R L} & S_{33}^{R L} & S_{34}^{R L} \\
S_{41}^{R L} & S_{43}^{R L} & S_{44}^{R L}
\end{array}\right]
$$

where

$$
\begin{gathered}
S_{11}^{R L}=S_{11}^{\prime}+S_{12}^{\prime} S_{21}^{\prime} / \operatorname{det}, S_{13}^{R L}=S_{13}^{\prime}+S_{12}^{\prime} S_{23}^{\prime} / \text { det, } S_{14}^{R L}=S_{14}^{\prime}+S_{12}^{\prime} S_{24}^{\prime} / \text { det, } \\
S_{31}^{R L}=S_{31}^{\prime}+S_{33}^{\prime} S_{21}^{\prime} / \text { det, } S_{33}^{R L}=S_{33}^{\prime}+S_{32}^{\prime} S_{23}^{\prime} / \text { det, } S_{34}^{R L}=S_{34}^{\prime}+S_{32}^{\prime} S_{24}^{\prime} / \text { det, } \\
S_{41}^{R L}=S_{41}^{\prime}+S_{42}^{\prime} S_{21}^{\prime} / \operatorname{det}, S_{43}^{R L}=S_{43}^{\prime}+S_{42}^{\prime} S_{23}^{\prime} / \text { det, } S_{44}^{R L}=S_{44}^{\prime}+S_{42}^{\prime} S_{24}^{\prime} / \text { det, } \\
\operatorname{det}=1-S_{22}^{\prime} .
\end{gathered}
$$

Through the latest equations, the elements of the scattering matrix $\left[S^{\prime}\right]$ related to the left reentrant coaxial section are used. In this case, the four-port representation of the left section (i.e., without the open-circuited regime at the port $2^{R L}$ of body " 1 ") is considered: 


$$
\left[S^{\prime}\right]=\left[\begin{array}{llll}
S_{11}^{\prime} & S_{12}^{\prime} & S_{13}^{\prime} & S^{\prime}{ }_{14} \\
S_{21}^{\prime} & S^{\prime}{ }_{22} & S^{\prime}{ }_{23} & S^{\prime}{ }_{24} \\
S^{\prime} & S_{32}^{\prime} & S^{\prime}{ }_{33} & S^{\prime}{ }_{34} \\
S^{\prime}{ }_{41} & S^{\prime}{ }_{42} & S^{\prime}{ }_{43} & S^{\prime}{ }_{44}
\end{array}\right]
$$

This matrix $\left[S^{\prime}\right]$ is formed by the conversion between it and the impedance matrix $\left[Z^{\prime}\right]$, as described in Reference [32]. Applying the approach described in Reference [28], as shown in Figure A1, it is possible to write the following equations for the matrix entirety:

$$
\left[Z^{\prime}\right]=\left[\begin{array}{cccc}
Z_{11}^{B L}+Z_{11}^{N L} & Z_{11}^{N L} & Z_{12}^{N L} & Z_{12}^{B L}+Z_{12}^{N L} \\
Z_{11}^{N L} & Z_{11}^{N L} & Z_{12}^{N L} & Z_{12}^{N L} \\
Z_{12}^{N L} & Z_{12}^{N L} & Z_{11}^{N L} & Z_{11}^{N L} \\
Z_{12}^{B L}+Z_{12}^{N L} & Z_{12}^{N L} & Z_{11}^{N L} & Z_{11}^{B L}+Z_{11}^{N L}
\end{array}\right],
$$

where

$$
\begin{gathered}
Z_{11}^{B L}=Z_{B L} \alpha_{B L}, Z_{12}^{B L}=Z_{B L} \beta_{B L}, Z_{11}^{N L}=Z_{N L} \alpha_{N L}, Z_{12}^{N L}=Z_{N L} \beta_{N L} \\
\alpha_{B L}=-j \cot \theta_{B L}, \beta_{B L}=-j \csc \theta_{B L}, \alpha_{N L}=-j \cot \theta_{N L}, \beta_{N L}=-j \csc \theta_{N L}, \\
\theta_{B L}=a_{B L}(\pi / 2)\left(f / f_{0 N L}\right), a_{B L}=\sqrt{\varepsilon_{r B L} / \varepsilon_{r N L}}, \theta_{N L}=(\pi / 2)\left(f / f_{0 N L}\right) .
\end{gathered}
$$

These quantities include: the characteristic impedance $Z_{B L}$ of the transmission line inside body " 1 " with dielectric filling $\varepsilon_{r B L}$; the characteristic impedance $Z_{N L}$ of the standalone body " 1 " placed inside the dielectric filling $\varepsilon_{r N L}$ without the internal transmission line (in other words, the body " 1 " is solid); $f$ and $f_{0 N L}$ are the current and reference frequencies of the body " 1 ", respectively, at which $\theta_{N L}=\pi / 2$.

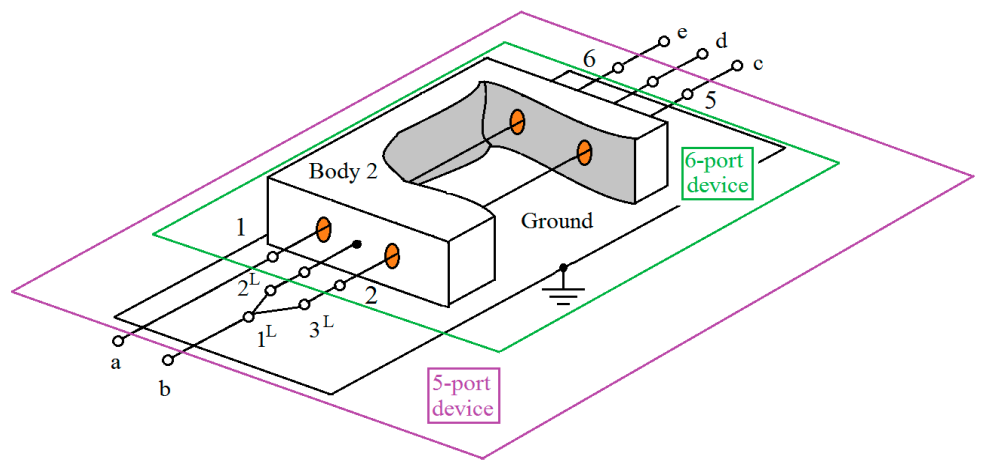

(a)

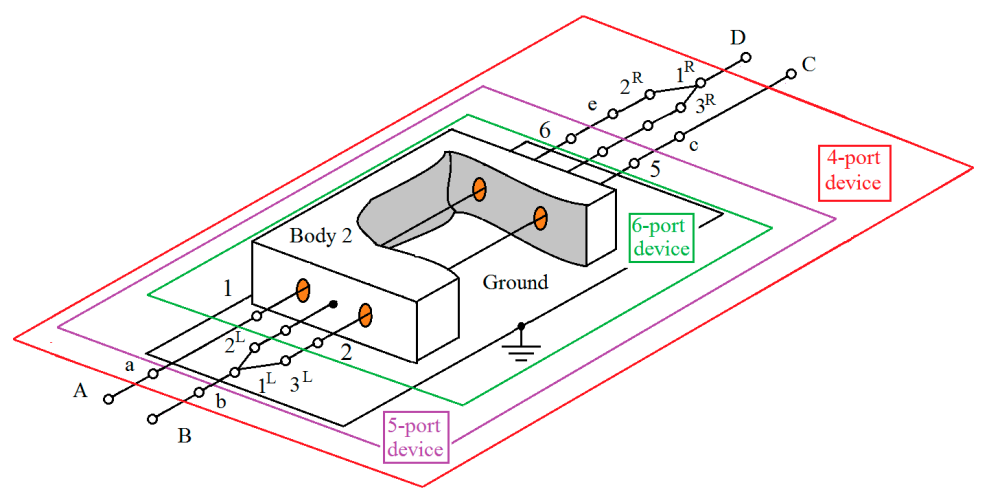

(b)

Figure A1. The six-port reentrant section on the base of body "2" placed above the ground: (a) after connection of the left three-port device, (b) after the connection of the right one. 
The latest representations are necessary to define the scattering matrix $\left[S^{3}\right]$ of the three-port network with port designations by numbers $1^{*}, 2^{*}$, and $3^{*}$, as shown in Figure A2a. Applying the technique described in Reference [33], one can write

$$
\left[S^{3}\right]=\left[\begin{array}{lll}
S_{11}^{3} & S_{12}^{3} & S_{13}^{3} \\
S_{21}^{3} & S_{22}^{3} & S_{23}^{3} \\
S_{31}^{3} & S_{32}^{3} & S_{33}^{3}
\end{array}\right]=\left[S_{P P}^{\prime \prime}\right]+\left[S_{P C}^{\prime \prime}\right]\left(\left[F^{\prime \prime}\right]-\left[S_{C C}^{\prime \prime}\right]\right)^{-1}\left[S_{C P}^{\prime \prime}\right]
$$

where

$$
\begin{aligned}
& {\left[S_{P P}^{\prime \prime}\right]=\left[\begin{array}{ccc}
S_{11}^{R L} & 0 & 0 \\
0 & S_{C C}^{4} & S_{C D}^{4} \\
0 & S_{D C}^{4} & S_{D D}^{4}
\end{array}\right],\left[S_{P C}^{\prime \prime}\right]=\left[\begin{array}{cccc}
S_{14}^{R L} & 0 & S_{13}^{R L} & 0 \\
0 & S_{C A}^{4} & 0 & S_{C B}^{4} \\
0 & S_{D A}^{4} & 0 & S_{D B}^{4}
\end{array}\right],} \\
& {\left[S_{C C}^{\prime \prime}\right]=\left[\begin{array}{cccc}
S_{44}^{R L} & 0 & S_{43}^{R L} & 0 \\
0 & S_{A A}^{4} & 0 & S_{A B}^{4} \\
S_{34}^{R L} & 0 & S_{33}^{R L} & 0 \\
0 & S_{B A}^{4} & 0 & S_{B B}^{4}
\end{array}\right],\left[S_{C P}^{\prime \prime}\right]=\left[\begin{array}{ccc}
S_{41}^{R L} & 0 & 0 \\
0 & S_{A C}^{4} & S_{A D}^{4} \\
S_{31}^{R L} & 0 & 0 \\
0 & S_{B C}^{4} & S_{B D}^{4}
\end{array}\right],\left[F^{\prime \prime}\right]=[F] .}
\end{aligned}
$$

The final stage of the consideration is the derivation of overall scattering matrix shown in Equation (4) in its definitive form. To do so, we should write the scattering matrix $\left[S^{R R}\right]$ of the right four-port reentrant coaxial section realized on the base of body " 3 ", as shown in Figure 2c. Through the current consideration, it is mandatory to use the open-circuited regime at the right body's port $3^{R R}$, as shown in Figure A2b. In other words, this port $3^{R R}$ may be omitted from a following analysis according to Reference [30]. Additionally, the approach described in Reference [30] should be modified in order to use all the other ports, namely: $1^{R R}, 2^{R R}$, and $4^{R R}$, as shown in Figure A2b. In accordance with the method described in Reference [35], one can write the scattering matrix $\left[S^{R R}\right]$ of the three-port device realized on the base of body " 3 " with open-circuited port $3^{R R}$ (in other words, it is omitted in Figure A2b):

$$
\left[S^{R R}\right]=\left[\begin{array}{lll}
S_{11}^{R R} & S_{12}^{R R} & S_{14}^{R R} \\
S_{21}^{R R} & S_{22}^{R R} & S_{24}^{R R} \\
S_{41}^{R R} & S_{42}^{R R} & S_{44}^{R R}
\end{array}\right]
$$

where

$$
\begin{gathered}
S_{11}^{R R}=S_{11}^{\prime \prime}+S_{13}^{\prime \prime} S_{31}^{\prime \prime} / \text { der, } S_{12}^{R R}=S_{12}^{\prime \prime}+S_{13}^{\prime \prime} S_{32}^{\prime \prime} / \text { der }, S_{14}^{R R}=S_{14}^{\prime \prime}+S_{13}^{\prime \prime} S_{24}^{\prime \prime} / \text { der }, \\
S_{21}^{R R}=S_{21}^{\prime \prime}+S_{23}^{\prime \prime} S_{31}^{\prime \prime} / \text { der, } S_{22}^{R R}=S_{22}^{\prime \prime}+S_{22}^{\prime \prime} S_{32}^{\prime \prime} / \text { der, } S_{24}^{R R}=S_{24}^{\prime \prime}+S_{22}^{\prime \prime} S_{34}^{\prime \prime} / \text { der }, \\
S_{41}^{R R}=S_{41}^{\prime \prime}+S_{43}^{\prime \prime} S_{31}^{\prime \prime} / \text { der }, S_{42}^{R R}=S_{42}^{\prime \prime}+S_{43}^{\prime \prime} S_{32}^{\prime \prime} / \text { der, } S_{44}^{R R}=S_{44}^{\prime \prime}+S_{43}^{\prime \prime} S_{34}^{\prime \prime} / \text { der }, \\
\text { der }=1-S_{33}^{\prime \prime} .
\end{gathered}
$$

Through the latest equations, the elements of the scattering matrix $\left[S^{\prime \prime}\right]$, related to the right reentrant coaxial section in the four-port representation, i.e., without open-circuited regime at the port $3^{R R}$ of body " 3 ":

$$
\left[S^{\prime \prime}\right]=\left[\begin{array}{llll}
S_{11}^{\prime \prime} & S_{12}^{\prime \prime} & S_{13}^{\prime \prime} & S_{14}^{\prime \prime} \\
S_{21}^{\prime \prime} & S_{22}^{\prime \prime} & S_{23}^{\prime \prime} & S_{24}^{\prime \prime} \\
S_{31}^{\prime \prime} & S_{32}^{\prime \prime} & S_{33}^{\prime \prime} & S_{34}^{\prime \prime} \\
S_{41}^{\prime \prime} & S_{42}^{\prime \prime} & S_{43}^{\prime \prime} & S_{44}^{\prime \prime}
\end{array}\right],
$$

are used. 


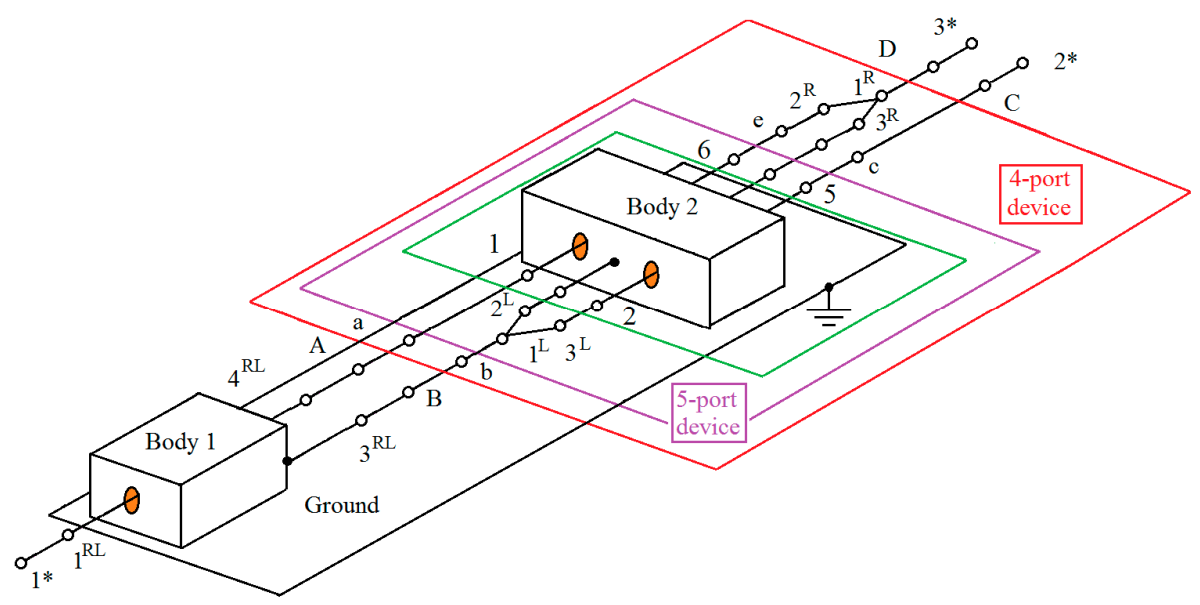

(a)

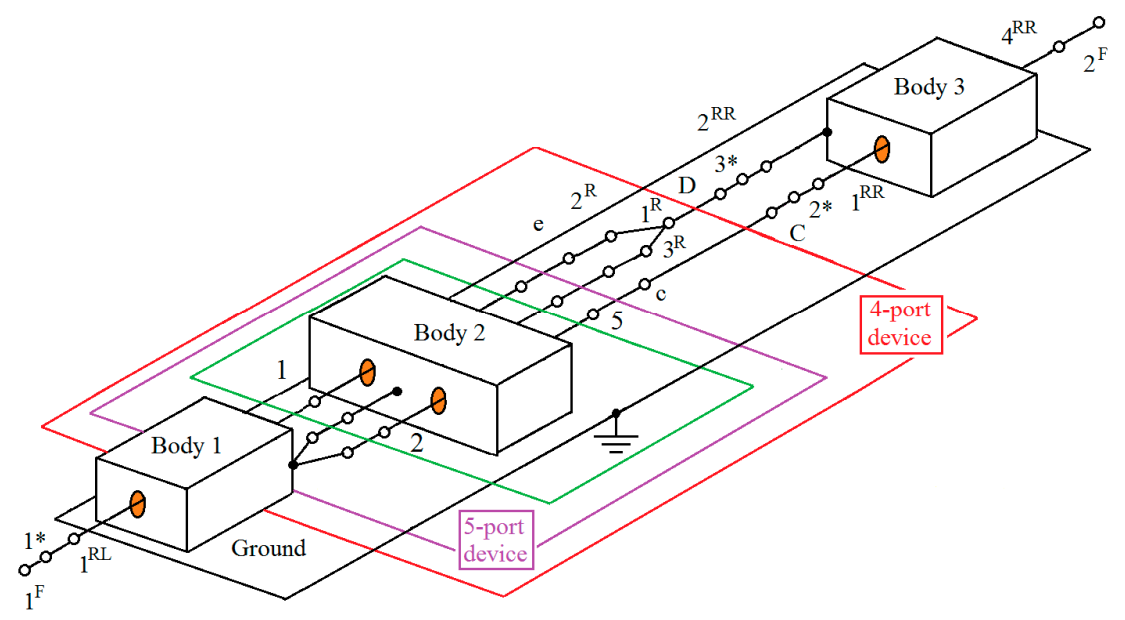

(b)

Figure A2. The reduced reentrant multi-ports related to the proposed filter: (a) after the connection of the left body " 1 ", (b) after connection of the right body "3", i.e., the proposed BSF itself.

On the analogy with the creation of the three-port device shown in Figure A2a, it is necessary to introduce the impedance matrix $\left[Z^{\prime \prime}\right]$ related to body " 3 " in its four-port representation. This matrix is the same as the one related to body " 1 " (i.e., $\left[Z^{\prime}\right]$ ), but the indices should be modified as follows:

$$
\left[Z^{\prime \prime}\right]=\left[\begin{array}{cccc}
Z_{11}^{B R}+Z_{11}^{N R} & Z_{11}^{N R} & Z_{12}^{N R} & Z_{12}^{B R}+Z_{12}^{N R} \\
Z_{11}^{N R} & Z_{11}^{N R} & Z_{12}^{N R} & Z_{12}^{N R} \\
Z_{12}^{N R} & Z_{12}^{N R} & Z_{11}^{N R} & Z_{11}^{N R} \\
Z_{12}^{B R}+Z_{12}^{N R} & Z_{12}^{N R} & Z_{11}^{N R} & Z_{11}^{B R}+Z_{11}^{N R}
\end{array}\right],
$$

where

$$
\begin{gathered}
Z_{11}^{B R}=Z_{B R} \alpha_{B R}, Z_{12}^{B R}=Z_{B R} \beta_{B R}, Z_{11}^{N R}=Z_{N R} \alpha_{N R}, Z_{12}^{N R}=Z_{N R} \beta_{N R}, \\
\alpha_{B R}=-j \cot \theta_{B R}, \beta_{B R}=-j \csc \theta_{B R}, \alpha_{N R}=-j \cot \theta_{N R}, \beta_{N R}=-j \csc \theta_{N R}, \\
\theta_{B R}=a_{B R}(\pi / 2)\left(f / f_{0 N R}\right), a_{B R}=\sqrt{\varepsilon_{r B R} / \varepsilon_{r N R}}, \theta_{N R}=(\pi / 2)\left(f / f_{0 N R}\right) .
\end{gathered}
$$

These equations include: the characteristic impedance $Z_{B R}$ of the transmission line inside body " 3 " with dielectric filling $\varepsilon_{r B R}$; the characteristic impedance $Z_{N R}$ of the standalone body " 3 " placed inside the dielectric filling $\varepsilon_{r N R}$ without the internal transmission line (i.e., body " 3 " is also solid); $f$ and $f_{0 N R}$ are the current and reference frequencies, respectively, of the body " 3 " at which $\theta_{N R}=\pi / 2$. 
Finally, we can define the overall scattering matrix $\left[S^{F}\right]$ that is used in the main text under Equation (4). Applying the technique described in Reference [33], the final equations for the proposed bandstop filter with the port designations by numbers $1^{F}$ and $2^{F}$, as shown in Figure A2b, are as follows:

$$
\left[S^{F}\right]=\left[\begin{array}{ll}
S_{11}^{F} & S_{12}^{F} \\
S_{12}^{F} & S_{11}^{F}
\end{array}\right]=\left[S_{P P}^{F}\right]+\left[S_{P C}^{F}\right]\left(\left[F^{F}\right]-\left[S_{C C}^{F}\right]\right)^{-1}\left[S_{C P}^{F}\right]
$$

where

$$
\begin{gathered}
{\left[S_{P P}^{F}\right]=\left[\begin{array}{cc}
S_{11}^{3} & 0 \\
0 & S_{44}^{R R}
\end{array}\right],\left[S_{P C}^{F}\right]=\left[\begin{array}{cccc}
S_{13}^{3} & 0 & S_{12}^{3} & 0 \\
0 & S_{42}^{R R} & 0 & S_{41}^{R R}
\end{array}\right],} \\
{\left[S_{C C}^{F}\right]=\left[\begin{array}{cccc}
S_{33}^{3} & 0 & S_{32}^{3} & 0 \\
0 & S_{22}^{R R} & 0 & S_{21}^{R R} \\
S_{23}^{3} & 0 & S_{22}^{3} & 0 \\
0 & S_{12}^{R R} & 0 & S_{11}^{R R}
\end{array}\right],} \\
{\left[S_{C P}^{F}\right]=\left[\begin{array}{cc}
S_{31}^{3} & 0 \\
0 & S_{24}^{R R} \\
S_{21}^{3} & 0 \\
0 & S_{14}^{R R}
\end{array}\right],\left[F^{F}\right]=[F] .}
\end{gathered}
$$

\section{References}

1. Hong, J.-S. Microstrip Filters for RF/Microwave Applications, 2rd ed.; Chang, K., Ed.; Wiley: Hoboken, NJ, USA, 2011.

2. Levy, R.; Cohn, S.B. A history of microwave filter research, design, and development. IEEE Trans. Microw. Theory Tech. 1984, 32, 1055-1067. [CrossRef]

3. Levy, R. Classic Works in RF Engineering, Volume 2, Microwave and RF Filters; Artech House: Norwood, MA, USA, 2007.

4. Mongia, R.K.; Bahl, I.J.; Bhartia, P.; Hong, J. RF and Microwave Coupled-Line Circuits; Artech House: Norwood, MA, USA, 2007.

5. Matthaei, G.L.; Young, L.; Jones, E.M.T. Microwave Filters, Impedance Matching Networks and Coupling Structures; McGraw-Hill: New York, NY, USA, 1964.

6. Hao, Z.-C.; Hong, J.-S. Ultrawideband filter technologies. IEEE Microw. Mag. 2010, 11, 56-68. [CrossRef]

7. Cameron, R.J.; Yu, M.; Wang, Y. Direct-coupled microwave filters with single and dual stopbands. IEEE Trans. Microw. Theory Tech. 2005, 53, 3288-3297. [CrossRef]

8. Levy, R.R.; Snyder, V.; Shin, S. Bandstop filters with extended upper passbands. IEEE Trans. Microw. Theory Tech. 2006, 54, 2503-2515. [CrossRef]

9. Shaman, H.; Hong, J.-S. Wideband bandstop filter with cross-coupling. IEEE Trans. Microw. Theory Tech. 2007, 55, 1780-1785. [CrossRef]

10. Chin, K.-S.; Yeh, J.-H.; Chao, S.-H. Compact dual-band bandstop filters using stepped-impedance resonators. IEEE Microw. Wireless Compon. Lett. 2007, 17, 849-851. [CrossRef]

11. Mandal, M.K.; Divyabramham, K.; Sanyal, S. Compact, wideband bandstop filters with sharp rejection characteristic. IEEE Microw. Wirel. Compon. Lett. 2008, 18, 665-667. [CrossRef]

12. Fathelbab, W.M. Two novel classes of band-reject filters realizing broad upper pass bandwidth-Synthesis and design. IEEE Trans. Microw. Theory Tech. 2011, 59, 250-259. [CrossRef]

13. Mandal, M.K.; Divyabramham, K.; Velidi, V.K. Compact wideband bandstop filter with five transmission zeros. IEEE Microw. Wirel. Compon. Lett. 2012, 22, 4-6. [CrossRef]

14. Xiao, J.-K.; Zhu, Y.-F. Multi-band bandstop filter using inner T-shaped defected microstrip. AEU Int. J. Electron. Commun. 2014, 68, 90-96. [CrossRef]

15. Atuchin, V.V.; Buhtiyarov, D.A.; Gorbachev, A.P. Compact printed microwave filters for wireless communication applications. Pac. Sci. Rev. A Nat. Sci. Eng. 2016, 18, 157-161. [CrossRef]

16. Koirala, G.R.; Shrestha, B.; Kim, N.-Y. Compact dual-wideband bandstop filter using a stub-enclosed stepped-impedance resonator. AEU Int. J. Electron. Commun. 2016, 70, 198-203. [CrossRef] 
17. Gupta, S.C.; Kumar, M.; Meena, R.S. Design \& analysis of a microstrip line multi band UWB filter. AEU Int. J. Electron. Commun. 2016, 70, 1556-1564.

18. Ebrahimi, A.; Withayachumnankul, W.; Al-Sarawi, S.F.; Abbott, D. Compact second-order bandstop filter based on dual-mode complementary split-ring resonator. IEEE Microw. Wirel. Compon. Lett. 2016, 26, 571-573. [CrossRef]

19. Min, X.; Zhang, H. Compact triple-band bandstop filter using folded, symmetric stepped-impedance resonators. AEU Int. J. Electron. Commun. 2017, 77, 105-111. [CrossRef]

20. Feng, W.J.; Hong, M.L.; Che, W.Q.; Xue, Q. Dual-band microstrip bandstop filter with multiple transmission poles using coupled lines. IEEE Microw. Wirel. Compon. Lett. 2017, 27, 236-238. [CrossRef]

21. Atuchin, V.V.; Gorbachev, A.P.; Khrustalev, V.A.; Tarasenko, N.V. Reentrant wideband quasi-elliptical bandpass filter. J. Electromagn. Waves Appl. 2018. [CrossRef]

22. Cohn, S.B. The re-entrant cross section and wide-band 3-dB hybrid couplers. IEEE Trans. Microw. Theory Tech. 1963, 11, 254-258. [CrossRef]

23. Lavendol, L.; Taub, J.J. Re-entrant directional coupler using strip transmission line. IEEE Trans. Microw. Theory Tech. 1965, 13, 700-701. [CrossRef]

24. Cristal, E.G. Re-entrant directional couplers having direct coupled center conductors. IEEE Trans. Microw. Theory Tech. 1966, 14, 207-208. [CrossRef]

25. Cristal, E.G. Nonsymmetrical coupled lines of reentrant cross section. IEEE Trans. Microw. Theory Tech. 1967, 15, 529-530. [CrossRef]

26. Cristal, E.G. Correction to "Nonsymmetrical coupled lines of reentrant cross section". IEEE Trans. Microw. Theory Tech. 1968, 16, 57. [CrossRef]

27. Gill, P.E.; Murray, W.; Wright, M.H. Practical Optimization; Academic Press: London, UK, 1981.

28. Gorbachev, A.P.; Potryasov, I.I. Designing microwave devices with shielded elements. J. Commun. Technol. Electron. 2000, 45, 462-466.

29. Gorbachev, A.P.; Tarasenko, N.V. The novel reentrant power splitters and bandstop elliptic filters. In Proceedings of the 2016 13th International Scientific-Technical Conference on Actual Problems of Electronics Instrument Engineering (APEIE), Novosibirsk, Russia, 3-6 October 2016; pp. 173-176.

30. Atuchin, V.V.; Gorbachev, A.P.; Khrustalev, V.A.; Tarasenko, N.V. The dual-band reentrant power splitter. AEU Int. J. Electron. Commun. 2018, 84, 21-26. [CrossRef]

31. Jones, E.M.T.; Bolljahn, J.T. Coupled-strip-transmission-line filters and directional couplers. IEEE Trans. Microw. Theory Tech. 1956, 4, 75-81. [CrossRef]

32. Frickey, D.A. Conversions between $S, Z, Y, h, A B C D$, and $T$ parameters which are valid for complex source and load impedances. IEEE Trans. Microw. Theory Tech. 1994, 42, 205-211. [CrossRef]

33. Cameron, R.J.; Kudsia, C.M.; Mansour, R.R. Microwave Filters for Communication Systems: Fundamentals, Design and Applications; Wiley: Hoboken, NJ, USA, 2007.

34. Otoshi, T.Y. On the scattering parameters of a reduced multiport. IEEE Trans. Microw. Theory Tech. 1969, 17, 722-724. [CrossRef]

35. Monaco, V.A.; Tiberio, P. Computer-aided analysis of microwave circuits. IEEE Trans. Microw. Theory Tech. 1974, 22, 249-263. [CrossRef]

36. Kolundzija, B.M.; Ognjanovic, J.S.; Sarkar, T.K. WIPL-D: Microwave Circuit and 3D EM Simulation for RF E Microwave Applications, Software and User's Manual; Artech House: Norwood, MA, USA, 2005.

37. Bakharev, S.I.; Volman, V.I.; Lib, Y.N.; Mamonova, N.; Muravtcov, A.; Sarkisjantc, A.; Silin, R.; Slavinsky, O.; Shirjaev, D. Handbook on Design of Microwave Circuits; Radio \& Swjaz: Moscow, Russia, 1982. (In Russian)

38. Alekseev, V.G.; Gridnev, V.N.; Nesterov, Y.I.; Filin, G. Technology of Digital Electronic Devices, Equipment and Automation; Wisshaya Shkola: Moscow, Russia, 1984. (In Russian)

39. Gorbachev, A.P. The reentrant wide-band directional filter. IEEE Trans. Microw. Theory Tech. 2002, 50, 2028-2031. [CrossRef] 
40. Gorbachev, A.P.; Malinko, D.A.; Vasilenko, A.A. The reentrant wideband bandpass/bandstop elliptic filters. In Proceedings of the 2010 10th International Conference on Actual Problems of Electronic Instrument Engineering APEIE-2010, Novosibirsk, Russia, 22-24 September 2010; pp. 56-63.

41. Gorbachev, A.P.; Churkin, V.S.; Vasilenko, A.A. The modified Schiffman phase shifter. In Proceedings of the 2010 10th International Conference on Actual Problems of Electronic Instrument Engineering APEIE-2010, Novosibirsk, Russia, 22-24 September 2010; pp. 124-126.

(C) 2019 by the authors. Licensee MDPI, Basel, Switzerland. This article is an open access article distributed under the terms and conditions of the Creative Commons Attribution (CC BY) license (http:/ / creativecommons.org/licenses/by/4.0/). 